\title{
PRINCIPLES OF DESIGN OF SPECIALIZED TECHNOLOGICAL EQUIPMENT
}

\author{
Oleksiy ROMANCHENKO \\ Volodymyr Dahl East Ukrainian National University, Department of Machinery Engineering and Applied \\ Mechanics, 59-a Central pr., Severodonetsk, 93400, Ukraine \\ e-mail: alexvromanchenko@snu.edu.ua
}

\begin{abstract}
The paper presents a universal principle of design of flexible schemes of specialized technological equipment and a modular principle of design of construction and elements of special technological equipment. The construction elements of equipment, which allow changing the linear dimensions of this equipment in a wide range are designed. The application of designed elements for changing one or several linear dimensions of the equipment is possible in a complex and independently of each other. The possibility of application of proposed design changes of equipment with the preservation of the required strength characteristics is substantiated. Three-dimensional models of construction elements and equipment in general are developed. Researches by changing of reservoir design by using a replaceable construction element are carried out. An analysis of the strength characteristics of integral reservoir and reservoir with a replaceable end wall from various materials is presented.
\end{abstract}

Keywords: specialized technological equipment, flexible schemes, modular principle, strength, constructions.

\section{FORMULATION OF THE PROBLEM}

The actual problem of machine-building industries is the expansion of the technological capabilities of specialized technological equipment (STE), designed for finishing treatment of parts. Finishing treatment is a next step after mechanical and heat treatment. The difficulties that arise at this stage of processing are due, on the one hand, to the wide range of processed parts, and on the other hand, to the choice of the treatment method. Parts can have different parameters of mass and dimensions, have a complex shape. Treatment methods, in turn, can impose restrictions on its capabilities, in particular, the overall dimensions of the part may not allow treatment on certain machine-tools or technological capabilities of the equipment do not allow achieving the required process performance by quantitative or qualitative indicators.

Vibration treatment technology consists in the fact that a working environment, parts and other intensifying additives are placed in a reservoir, which can have various shapes and volumes. Parts can be placed freely or be fixed into the reservoir. Further, oscillatory movements are imparted to the reservoir with the help of one or several external energy sources. As a result of the relative movement and mutual pressure of the granules of working medium and the parts, the processes of microcutting and elastoplastic deformation occur, which ensures the removal of the defective metal layer, as well as a decrease in the surface roughness of parts. The vibration processing process is dimensionless, it does not change the shape and size of the parts [1,2].

The main competitive advantage of the method is the ability to simultaneously treat a large number of parts of various shapes and dimensions. However, the main factor limiting the versatility of such equipment is the volume and mass of the reservoir [3]. Obviously, the volume of the reservoir depends on its linear dimensions, which in turn predetermine the overall dimensions of all equipment. As a rule, when designing of vibration equipment, the principles of unification and interchangeability of equipment elements are applied, which makes it possible to reduce the nomenclature of parts required for the production of this equipment, as well as to quickly replace worn-out elements. But, the design of most of the known machine-tools does not provide for the use of a reservoir with increased or reduced overall dimensions, as well as several sequentially installed reservoirs.

To solve the problems described above, when modernizing existing equipment and synthesizing new equipment, it is necessary to apply the "principle of design of flexible schemes of specialized technological equipment" - the design of the equipment must ensure a change in the linear dimensions of its elements. It is important to note that this principle is not narrowly focused, its application is possible and necessary for all classes and types of specialized technological equipment.

(C) 2022 by the Authors. Licensee Polish Society of Technical Diagnostics (Warsow. Poland). This article is an open access article distributed under the terms and conditions of the Creative Commons Attribution (CC BY) license http://creativecommons.org/licenses/by/4.0/). 
The possibility of implementing flexible equipment schemes also requires the use of a "modular principle of design of construction and elements of special technological equipment":

- the general design of specialized technological equipment should consist of a larger number of assembly parts while maintaining the strength characteristics;

- parts included in assembly units must have simple geometric shapes.

The implementation of the modular principle makes the stage of equipment designing more complicated, requires a more detailed study of the elements and the entire structure, but allows laying down a huge potential for expanding the technological capabilities of parts processing. It is even more difficult to implement this principle in the production of elements on classical metal cutting equipment, since each element is quite expensive and requires separate adjustment.

However, the use of new technologies makes it possible to solve these problems. Such technologies, for example, include additive manufacturing, in which the creation of a part occurs by sequential application of layers of material.

\section{ANALYSIS OF RESEARCHES AND PUBLICATIONS}

Vibration treatment is a relatively modern finishing method. The first fundamental works on this topic date back to the 60 s of the 20th century [4, 5 , 6], it was during this period that the mass introduction of vibration equipment into production began.

The vibration treatment process is complex and depends on many factors. The number of factors and the degree of their influence on the performance of the process is the subject of many studies $[1,2,4]$. Based on the analysis of previous studies in works $[5,6]$, the main factors that have a direct impact on obtaining the technological effect of vibration processing were formulated:

1) the initial state of the workpiece and the final purpose of treatment (production method, material, physical and mechanical properties of the workpiece, state of the surface layer, shape and dimensions of the part);

2) modes of movement (the nature of reservoir oscillations, trajectories of its movement, amplitude and frequency of oscillations);

3) design features of the reservoir (shape, volume of the reservoir, availability of devices);

4) working medium (abrasive granules with certain characteristics, the presence of liquid solutions);

5) chemical, electrochemical, electrophysical intensification of the vibration treatment process;

6) design features of the vibration machine;

7) mechanization and automation of auxiliary operations, such as loading, unloading, separation of workpieces and abrasive granules.
Each factor in itself is a subject that requires separate in-depth research, which is reflected in the works of many authors.

Since in the future, the vibration method began to treat parts that had not been previously treated, as well as parts that combined the features of several groups. Studies have appeared that require upgrade of the classifier presented above, and the introduction of subgroups of details. Such parts, for example, include long parts, since they can combine features characteristic of both medium-sized parts (in cross-section, a part can be comparable to the size of granules or exceed them), and for large parts (linear dimensions of a part can significantly exceed the width of reservoir and be comparable to the length of the reservoir in one of the sizes) [7].

The search for a mathematical model that most fully describes the nature of movement of working medium in an oscillating reservoir remains a particularly difficult and not fully solved problem. There are many works devoted to this topic $[8,9,10$, $11,12]$. It should be noted that early studies were mainly carried out for U-shaped reservoirs, but even such experimental conditions as modern modeling using the finite element method did not allow to come to unambiguous conclusions [13]. Today, the solution to this problem has become even more urgent and complex, since widely applied machinetools with a toroidal shape of a reservoir, as well as equipment with a multi-energy effect on the working medium which can combine several independent vibrational sources and create complex trajectories of movement of details [14]. One more type of free abrasive treatment should also be singled out separately - vibration hardening treatment. This type of treatment is used in high-tech industries, especially in the aerospace industry, where, thanks to the introduction of this technology, the strength and performance characteristics of parts of high precision and complexity are significantly increased $[15,16,17]$.

Another way to improve the performance of the method is development of additional devices for vibration equipment, while their design features can be radically different depending on their purpose. According to their purpose, additional devices for vibration equipment can be divided into 3 groups:

1. Devices for mechanization and automation of auxiliary operations, such as loading, unloading, separation of workpieces and abrasive granules $[18,19]$.

2. Devices for fixing the part. In this case, the part can be fixed motionlessly or with the ability to move within predetermined limits, and also the part can be forcibly communicated with additional movement [13].

3. Devices for intensifying the treatment process. These include various deflectors for influencing the direction of movement of the working medium, orientation of freely moving parts, etc. [8]. 
All of the above must be considered when designing finishing equipment. The issues of arrangement and design of schemes of technological equipment, as a rule, are considered in detail in the context of individual elements [20, 21, 22]. The schemes with several independent reservoirs, as well as with a large number of oscillation sources, have not been fully investigated. Special attention should be paid to the issues of control and management of the electric drive [23, 24].

However, solving this problem obviously requires an integrated approach. The synthesis of new equipment should be carried out according to clearly defined principles. Recently, works devoted to this topic have begun to appear [25, 26, 27, 28], but it is obvious that this issue is relevant and requires further in-depth research.

Objective: Study of the principles of design of specialized technological equipment.

Research objectives:

Development of structural elements of vibration equipment for treatment of small parts using the principle of design of flexible schemes of specialized technological equipment and a modular principle of design of construction and elements of special technological equipment.

Comparative analysis of the design and strength characteristics of the developed elements.

\section{PRESENTATION OF THE MAIN MATERIAL}

\subsection{Study of the effect of use of a replaceable end wall on the strength characteristics of reservoir}

The designed equipment must have maximum universality. One of the effective ways to expand its technological capabilities is the use of a removable end wall. The presence of two interchangeable end walls makes it possible to use the treatment of parts by through method, i.e. treat parts with linear dimensions that exceed the longitudinal dimensions of the reservoir. In addition, it becomes possible to quickly mount walls with specialized fixtures or non-standard lining, and loading and unloading parts and working medium can also be simplified. It is interesting to use a transparent wall for visual assessment of the treatment process, estimation of the movement character of abrasive granules and the trajectory of the parts. For this purpose, an end wall made of transparent acrylic is installed. Acrylic differs in its characteristics from the initial material of the reservoir - PET plastic. To check the effect of end wall material on the strength characteristics, a simulation was carried out using the finite element method with linear analysis. It is also important to evaluate the effect of having a replaceable end wall on the strength of reservoir.

From the analysis of the simulation results presented in Figures $1-3$ and Table 1, it is obvious that the presence of a replaceable end wall affects the strength of the reservoir. Compared to a one-piece reservoir, a reservoir with a PET end wall has increased maximum tensions and deformations values by $27 \%$ and $10 \%$, respectively.

Interestingly, the maximum value of displacement index changed by less than $1 \%$, moreover, the best performance was shown by the container with a replaceable end wall. It is important to note that despite the difference in the absolute indicators of strength characteristics, the overall picture of the distribution of loads has the same character. The critical points of tensions, displacements and deformations are not in the place of the replaceable wall, therefore, the use of a replaceable end wall is advisable, since the decrease in strength characteristics is not significant, and its presence expands the technological capabilities of the method.

The change in the material of the end wall has practically no effect on the strength characteristics.

\subsection{Application of the principle of design of flexible schemes of specialized technological equipment and a modular principle of design of construction and elements of special technological equipment}

The application of the principle of a flexible scheme of specialized technological equipment is possible when designing equipment of a modular type, which was laid down at the stage of synthesis of the machine-tool developed in this work. So, special attachment points of reservoir - "lugs" with which it is attached to the frame were provided in the design of the machine-tool. This solution allows quick replacement of the reservoir. A quick change of the reservoir is important, because production of a reservoir using additive technologies is quite cheap and does not take much time. This type of fastening of reservoir differs from the traditional one, in which it is usually rigidly connected to the frame by springs. The reservoir itself equipped by a replaceable end wall.

The original frame structure was one-piece (Fig. 4a). Implementing the modular principle, its design has been divided into 8 parts. The frame is assembled using bolted connections. This design made it possible to change the linear dimensions by introducing minor design changes - changing parts of the equipment by additional elements or making new ones (Fig. 4b).

Changing the height of the frame is provided by changing the linear dimension $h$ of the "bracket" part (4 units) along the machine-tool axis z (Fig. 5a). It is also possible to change the dimension $h$ by using additional "inserts" (Fig. 5b).

Changing the width of the frame is provided by changing the linear dimension $w$ of the "base" part ( 2 units) along the machine-tool axis y (Fig. 6). The second method is the use of expansion "inserts" with stiffening ribs for the bracket parts, while the "inserts" can simultaneously adjust the dimension $h$ (Fig. 7). To ensure the required rigidity, "inserts" can be connected with additional amplifiers (Fig. 8). 


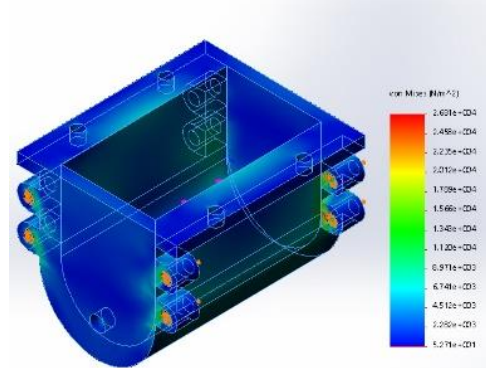

a)

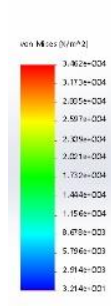

b)

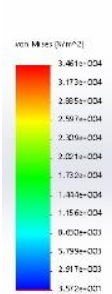

c)

Fig. 1. Reservoir tensions: a) without end wall; b) with an end wall; c) with an end wall made of acrylic

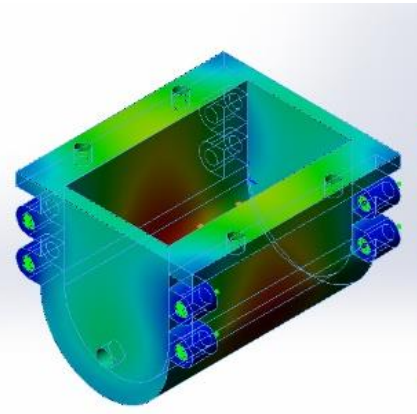

a)

a)

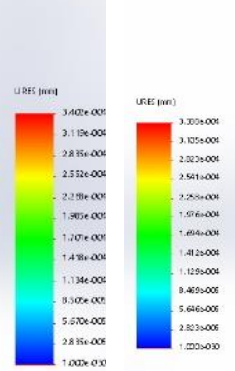

tomeos

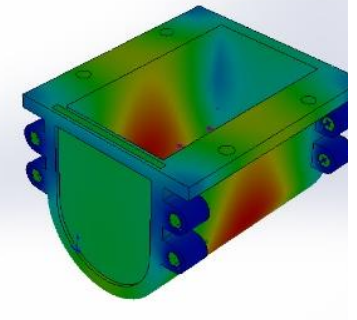

b)

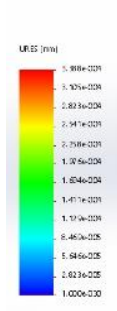

c)

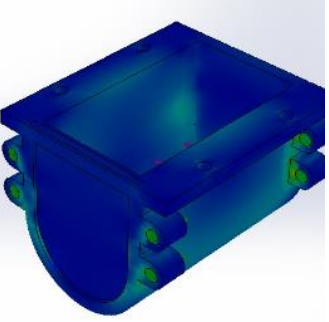

F. 2. Reservoir displacements: a) without end wall; b) with an end wall; c) with an end wall made of acrylic

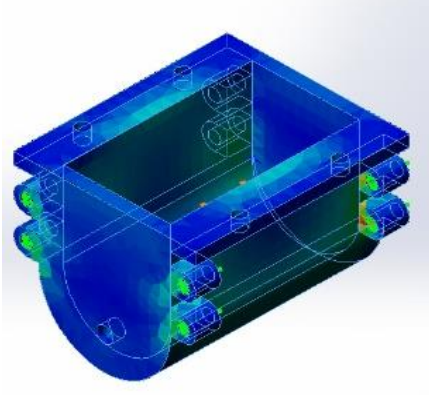

a)

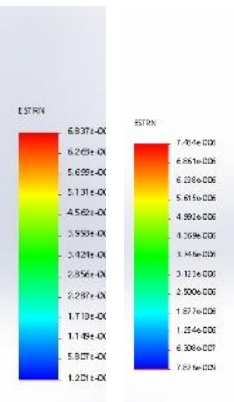

)

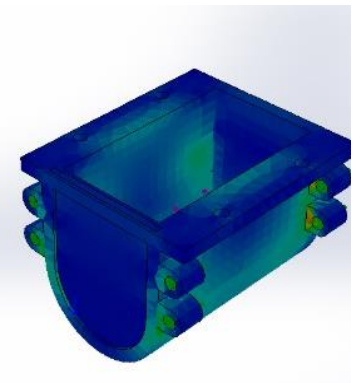

b)

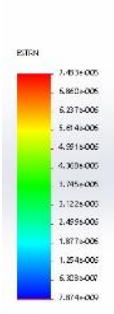

c)

Fig. 3. Reservoir deformation: a) without end wall; b) with an end wall; c) with an end wall made of acrylic

Table 1

Indicators of tensions, displacements deformations of reservoir with and without a replaceable end wall.

\begin{tabular}{|c|c|c|c|}
\hline \multirow[b]{2}{*}{ Strength characteristics } & \multicolumn{3}{|c|}{ Type of reservoir } \\
\hline & One-piece & $\begin{array}{c}\text { With replaceable PET } \\
\text { plastic wall }\end{array}$ & $\begin{array}{l}\text { With replaceable acrylic } \\
\text { wall }\end{array}$ \\
\hline \multirow{2}{*}{$\begin{array}{l}\text { Tensions, } \\
\text { vonMises }\left(N / m^{\wedge} 2\right)\end{array}$} & $\max .2 .681 e+004$ & $\max .3 .462 e+004$ & $\max .3 .461 e+004$ \\
\hline & $\min .5 .271 e+001$ & $\min .3 .214 e+001$ & $\min .3 .572 e+001$ \\
\hline \multirow{2}{*}{$\begin{array}{l}\text { Displacements, } \\
\text { URES (mm) }\end{array}$} & $\max .3,402 e-004$ & $\max .3,388 e-004$ & $\max .3,388 e-004$ \\
\hline & $\min .1,000 e-030$ & $\min .1,000 e-030$ & $\min .1,000 e-030$ \\
\hline \multirow{2}{*}{$\begin{array}{l}\text { Deformations, } \\
\text { ESTRN }\end{array}$} & $\max .6 .837 e-006$ & $\max .7,484 e-006$ & $\max .7,483 e-006$ \\
\hline & $\min .1 .201 e-008$ & $\min .7 .876 e-009$ & $\min .7 .874 e-009$ \\
\hline
\end{tabular}




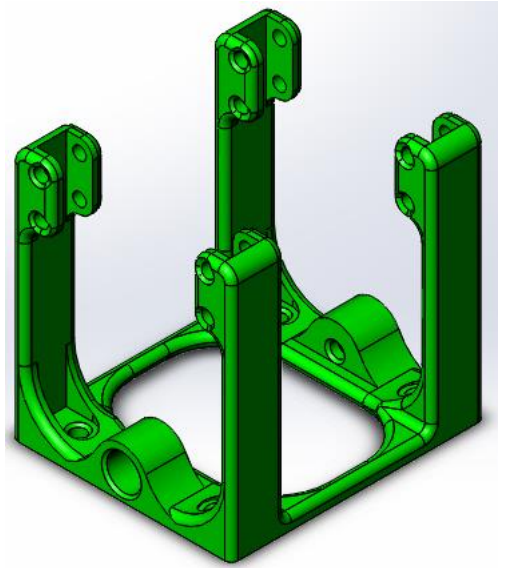

a)

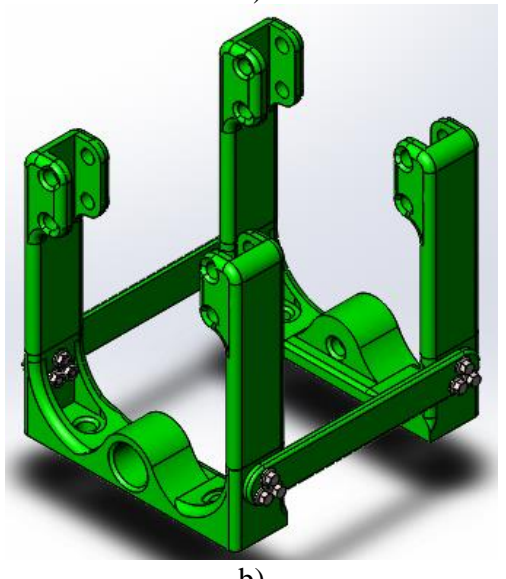

b)

Fig. 4. Construction of one-piece frame of vibration equipment: a) one-piece;

b) assembled

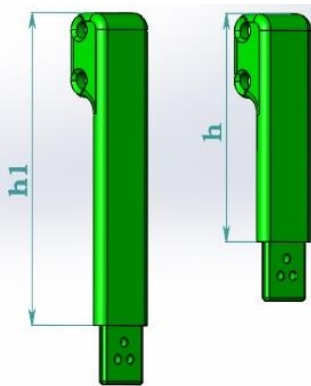

a)

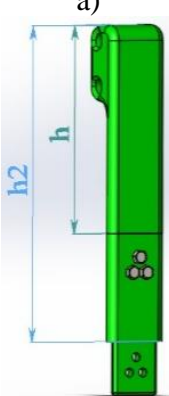

b)

Fig. 5. Frame bracket: a) with variable dimension $\mathrm{h}$; b) with an additional element for changing of dimension $\mathrm{h}$

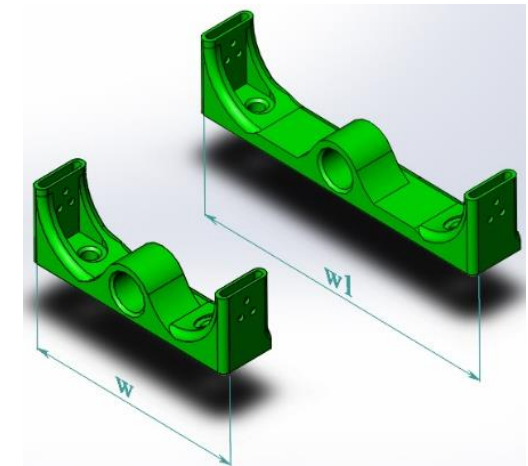

Fig. 6. Base of frame with changing of dimension w

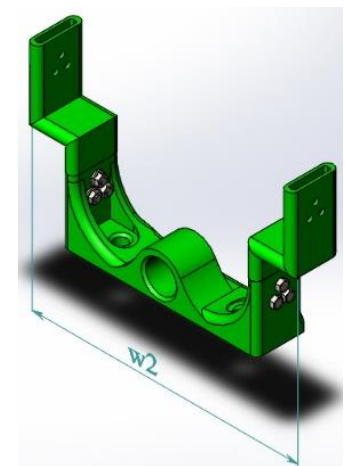

Fig. 7. Frame with additional elements for changing of dimension $\mathrm{w}$

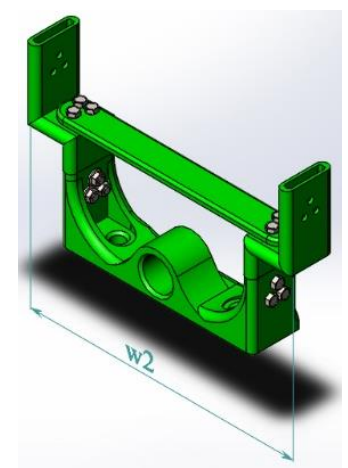

Fig. 8. Inserts with additional stiffening reinforcement for changing of dimensions $w$ and $h$

Adjustment of the frame along the length is carried out by changing the linear dimension 1 of the "crossbar" part connecting the frame base along the machine-tool axis x (Fig. 9).

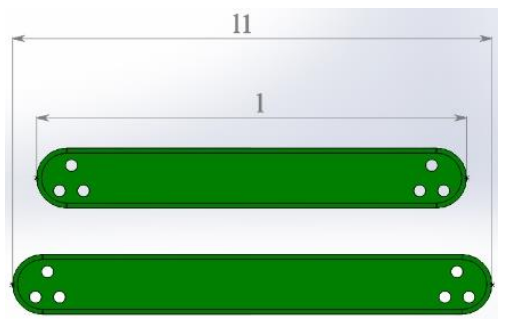

Fig. 9. Connecting crossbar for changing of dimension 1 
Thus, following the principle of creating a flexible scheme of specialized technological equipment and the modular principle, the presented machine-tool allows to change the linear dimensions along three coordinate axes by minor changes in elements or the use of new parts.

The general scheme of equipment with variable elements is shown in Figure 10.

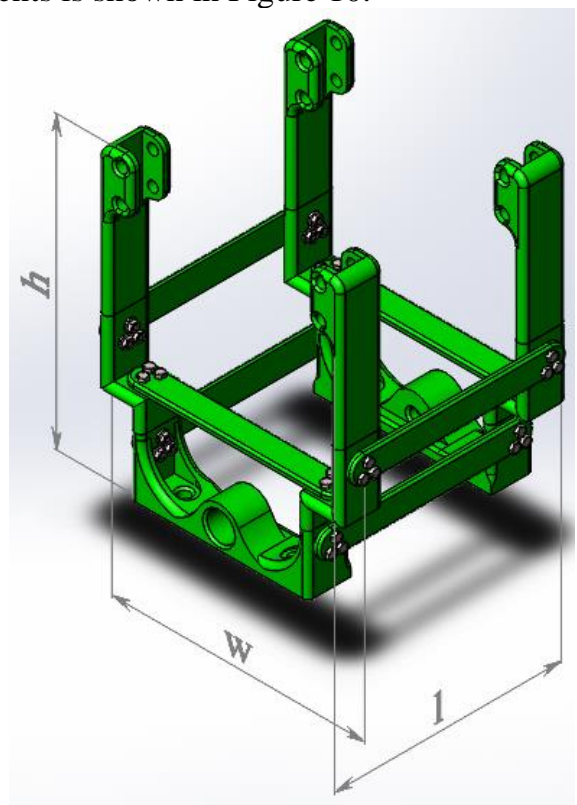

Fig. 10. Frame of vibration equipment with the ability to change the linear dimensions along three coordinate axes

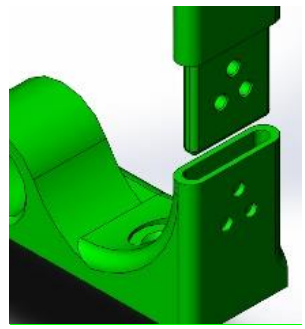

a)

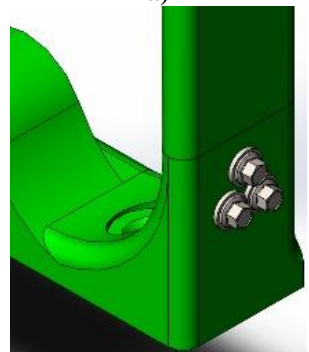

b)

Fig. 11. Scheme of elements connection:

a) groove with holes; b) bolted connection

It is important to note that accurate positioning is ensured by designing elements with grooves and holes (Fig. 11, a). Fast assembly and installation, as well as the required rigidity is ensured by bolted connections (Fig. 11, b).

As a result of the experimental research using 3D printing technology, equipment for the finishing vibration treatment of parts of the jewelry industry was produced, which is shown in Figure 12.

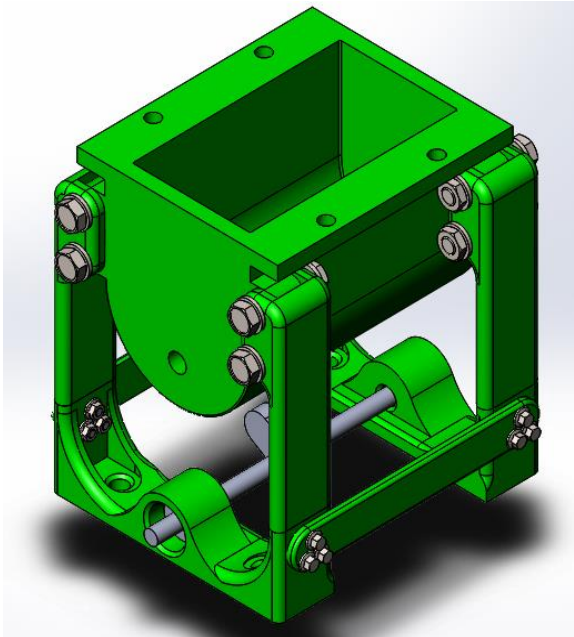

Fig. 12. Equipment for finishing vibration treatment of jewelry industry parts

\section{CONCLUSIONS}

As a result of research of designing of specialized technological equipment for vibration finishing of parts, the possibility and the expediency of use the principle of design of a flexible schemes of specialized technological equipment and modular principle at the stage of equipment synthesis was established.

It has been established that the presence of a replaceable end wall of the reservoir has an insignificant effect on the strength characteristics while maintaining the general character of loads distribution and is expedient, since it significantly expands the technological capabilities of the equipment. The material of the removable wall does not affect the strength characteristics.

Design solutions that allow changing the linear dimensions of the equipment in a wide range both in combination and independently of each other have been proposed.

Based on the obtained data, a machine-tool for vibration finishing of small parts was designed and manufactured by the additive method.

Declaration of competing interest: The author declares no conflict of interest.

\section{REFERENCES}

1. Babichev AP. Fundamentals of vibration technology. Rostov-on-Don: DSTU Publishing Center; 2008.

2. Kartashov IN, et al. Treatment of parts by free abrasives in vibrating reservoirs. Kiev: Highest school; 1975.

3. Romanchenko OV. Technological process of manufacturing of reservoir of vibrational machine-tool from composite materials. International scientific journal Technological audit and production reserves. 2015; 6/3 (26): 31-37.

4. Sankov NN, Zibenberg AN. Mechanization and automation of abrasive treatment of castings. Moscow: Mechanical Engineering; 1972.

5. Ventskevich G. Zh. The influence of some parameters of an abrasive filler on the efficiency of the grinding 
process in vibrating reservoirs [dissertation]. Odessa, SU; 1986.

6. Mitsyk V.Ya. Intensification of parts treatment in vibrating reservoirs by counter-moving flows of the working medium [dissertation]. Voroshilovgrad; SU; 1986.

7. Romanchenko OV. Expansion of technological capabilities of vibration equipment by creating conditions for treatment of long parts: [dissertation]. Chernigiv; UA; 2011.

8. Blekhman II, Janelidze G.Yu. Vibration displacement. Moscow: Nauka; 1964.

9. Kopylov Yu.R. Vibration impact hardening. Voronezh: Voronezh Institute of the Ministry of Internal Affairs of Russia; 1999.

10. Lubenskaya LM. Features of energy processes in deformable working medium when treatment of parts in vibrating reservoirs: [dissertation]. Lugansk; UA; 1992.

11. Ciampini D, Papini M, Spelt JK. Impact velocity measurement of media in a vibratory finisher. Journal of Materials Processing Technology. 2007;183:347357.

https://doi.org/10.1016/j.jmatprotec.2006.10.024.

12. Barletta M, Rubino G, Valentini PP. Experimental investigation and modeling of fluidized bed assisted drag finishing according to the theory of localization of plastic deformation and energy absorption. Int $\mathbf{J}$ Adv Manuf Technol. 2015;77: 2165-2180. https://doi.org/10.1007/s00170-014-6620-y.

13. Kang YS, Hashimoto F, Johnson SP, Rhodes JP. Discrete element modeling of 3D media motion in vibratory finishing process. CIRP Annals Manufacturing Technology. 2017;66(1):313-316. https://doi.org/10.1016/j.cirp.2017.04.092.

14. Mitsyk AV, Fedorovich VA. A new type of finishing and stripping method and an aggregated vibrating machine of vibration and centrifugal dynamic action. Cutting and tools in technological systems. 2016; 86: 96-102.

15. Babichev AP, Pastukhov FA, Motrenko PD, Chuchukalov AP. Analysis of technological schemes of vibration impact hardening treatment of long parts. Hardening technologies and coatings. Mechanical hardening treatment. 2006;5:3-5.

16. Davidson DA. Surface finishing reaches new heights: Mass media finishing techniques can improve aircraft part performance and service life. Metal finishing. 2005;103(3): 25-28.

17. Sangid MD, Stori JA, Ferriera PM. Process characterization of vibrostrengthening and application to fatigue enhancement of aluminum aerospace components - part I: Experimental study of process parameters. Int J Adv Manuf. Technol. 2011;53: 545560.

18. Babichev AP. editor. The use of vibration technologies in the operations of finishing and cleaning treatment of parts (cleaning, washing, removal of burrs, edge treatment). Rostov-on-Don: DSTU; 2010.

19. Romancheno OV, Romanchenko Yu.A. Review of devices for auxiliary operations of vibration Treatment. Vinyk of Volodymyr Dahl East Ukrainian National University. 2016; (226): 76-86.

20. Krol O, Sokolov V, Tsankov P. Modeling of vertical spindle head for machining center. Journal of Physics: Conference Series. 2020;1553:012012. https://doi.org/10.1088/1742-6596/1553/1/012012.
21. Krol O, Sokolov V. Modeling of spindle node dynamics sing the spectral analysis method. In: Advances in Design, Simulation and Manufacturing III. DSMIE 2020. Lecture Notes in Mechanical Engineering. 2020;1:35-44. https://doi.org/10.1007/978-3-030-50794-7 4.

22. Krol O, Porkuian O, Sokolov V, Tsankov P. Vibration stability of spindle nodes in the zone of tool equipment optimal parameters. Comptes rendus de l'Acade'mie bulgare des Sciences. 2019; 72(11): 1546-1556. https://doi.org/10.7546/CRABS.2019.11.12.

23. Sokolov V, Porkuian O, Krol O, Baturin Y. Design calculation of electrohydraulic servo drive for technological equipment. In: Advances in Design, Simulation and Manufacturing III. DSMIE 2020. Lecture Notes in Mechanical Engineering. 2020;1: 7584. https://doi.org/10.1007/978-3-030-50794-7 8.

24. Sokolov V, Porkuian O, Krol O, Stepanova O. Design calculation of automatic rotary motion electrohydraulic drive for technological equipment. in: advances in design. Simulation and Manufacturing IV. DSMIE 2021. Lecture Notes in Mechanical Engineering. 2021;1:133-142. https://doi.org/10.1007/978-3-030-77719-7_14.

25. Mitsyk AV, Fedorovich VA. Analytics, comments and classification of finishing and cleaning vibration treatment technologies, created by combining various schemes of energy impact. Modern technologies in machinery. NTU "KhPI". 2016;1: 175-179.

26. Kotliar A, Basova Y, Ivanov V. et all. Ensuring the economic efficiency of enterprises by multi-criteria selection of the optimal manufacturing process. Management and Production Engineering Review. 2020;11(1):52-61. https://doi.org/10.24425/mper.2020.132943.

27. Mitsyk AV. Multi-energy technologies and modular equipment in the practice of finishing and cleaning vibration treatment of parts with free abrasive mediim. Vinyk of Volodymyr Dahl East Ukrainian National University. 2015; 5(222): 64-72.

28. Kalmykov MA, Romanchenko OV. Determination of the functional dependence between the amplitude of the container and its length. Eastern-European Journal of enterprise technologies. 2011; 3/7:19-23.

Received 2021-12-19

Accepted 2022-02-17

Available online 2022-02-18

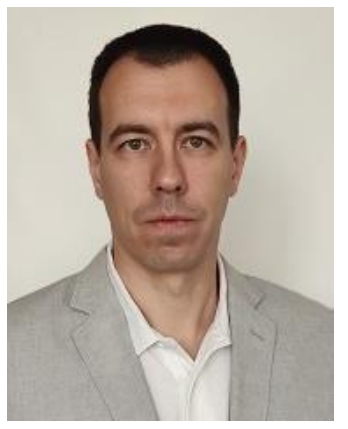

Oleksiy ROMANCHENKO (ORCID 0000-0002-43271105), PhD, Assistant Professor of the Machinery engineering and applied mechanics department of the Volodymyr Dahl East Ukrainian National University, Severodonetsk, Ukraine. Main directions of scientific research: machinery engineering, applied mechanics, special technological equipment. 